\title{
L'évolution du droit au développement devant les juridictions et quasi-juridictions régionales africaines
}

\section{Enguerrand Serrurier}

\section{MOTS-CLÉS}

Droit au développement, droits de l'homme, Charte africaine des droits de l'homme et des peuples.

\section{RÉSUMÉ}

Le droit au développement passe pour une notion quelque peu oubliée, souvent caricaturée, et apparemment décevante dans sa concrétisation depuis sa reconnaissance consensuelle lors de la Conférence mondiale sur les droits de l'homme de 1993. Tout n'a pourtant pas été dit et ce concept connaît ces dernières années des progrès discrets mais notables, qui s'expriment sur le terrain contentieux sous l'impulsion des juridictions et quasi-juridictions africaines. 


\section{Introduction}

« 'Histoire est le récit des choses advenues ; la Gazette, seulement le bruit qui en court » (Renaudot, 1632), et l'édition du 19 septembre 2014 de la Kenya Gazette (Presidency of Kenya, 2014) semble bien confirmer l'ancienne sentence en se faisant l'écho d'une décision historique de la Commission africaine des droits de l'homme et des peuples, rendue fin 2009, Endorois c. Kenya (Commission ADHP, 2009c). En effet, en informant le public de la création d'un groupe de travail interministériel par décret présidentiel, le journal officiel kényan atteste enfin des débuts de la mise en ouvre de cette sentence de la quasi-juridiction ${ }^{1}$ régionale africaine. Car l'affaire des Endorois a abouti, pour la première fois dans le système africain des droits de l'homme, à la sanction effective de la violation du droit au développement d'une tribu traditionnelle de pasteurs chassés de leurs terres ancestrales, suite à une expulsion opérée trente ans auparavant au profit d'un complexe hôtelier et touristique. Le cas paraît quelque peu anecdotique, mais il est remarquable par ses effets théoriques et opérationnels : un État africain entend se conformer aux prescriptions d'une instance internationale de protection des droits de l'homme, concernant la violation d'un droit que certains décrivaient jadis comme une fantasmagorie (Donnelly, 1985). Nombre de clichés éculés sont ici combattus et, d'un point de vue juridique, c'est le renforcement du droit au développement qui en constitue l'élément le plus notable.

Bien que l'événement eût été en vérité assez inattendu, bien des espoirs pouvaient naître du succinct article 22 de la Charte africaine des droits de l'homme et des peuples adoptée à Banjul (Gambie) en 1981 et entrée en

1 Par " quasi-juridiction ", il est entendu, en droit international, un organe qui n'est pas formellement un tribunal mais qui rend des décisions en appliquant le droit après une procédure contradictoire similaire, ce qui rend l'ensemble assez similaire sur le fond à ce en quoi consiste la tâche ordinaire des juges. Cette dénomination désigne des instances qui sont aptes à recevoir des réclamations portant sur un litige juridique telles que les diverses commissions d'experts des Nations unies, l'Organe de règlement des différends de I'Organisation mondiale du commerce, ou encore, sur le continent noir, la Commission africaine des droits de l'homme et des peuples (Commission ADHP). La distinction avec une véritable juridiction, au sens classique, est parfois subtile et mouvante (Santulli, 2000) car les deux types d'instance assument à des degrés variés une fonction juridictionnelle, même si dans le cas des quasi-juridictions celle-ci peut se colorer d'une volonté de négociation politique. 
vigueur en 1986 (OUA, 1981), applicable dans tous les États membres de l'Union africaine. Cette stipulation conventionnelle reconnaît en son paragraphe premier que «tous les peuples ont droit au développement économique, social et culturel, dans le respect de leur liberté et de leur identité » et fait peser en son paragraphe second la responsabilité de sa réalisation sur « les États, séparément ou en coopération ».

Sans application contentieuse, cet article 22 se serait limité à témoigner d'un débat appartenant peut-être à l'histoire du droit international, puisque le constat d'échec est devenu une banalité d'usage dès lors qu'est évoqué le droit au développement ${ }^{2}$. Néanmoins, en l'actionnant enfin par le biais de leur jurisprudence récente, les juridictions internationales africaines ont réanimé le droit au développement et œuvrent à sa complétion. L'effet n'en est pas négligeable car la démarche adoptée est celle d'un véritable échange juridique où le juge africain s'approprie et réinterprète les différentes normes internationales du développement à travers un éclectisme fécond. L'intérêt de l'analyse de la jurisprudence de l'article 22 de la Charte africaine embrasse, au-delà du cadre régional, le droit international général, le droit international des droits de l'homme et le droit des investissements, puisque ce droit pose des garanties fondamentales pour les individus et les populations dans le processus de développement, en matière économique, sociale, culturelle et foncière - peut-être transposables dans d'autres contextes. Au-delà des juristes et vu le caractère transdisciplinaire du développement, l'émergence d'un droit subjectif en la matière peut aussi, sans doute, intéresser les autres sciences sociales concernées par ce champ de réflexion. De plus, cette construction jurisprudentielle n'a guère suscité de commentaires en langue française ${ }^{3}$, à l'inverse de la doctrine anglophone qui s'est montrée plus prolixe sur le sujet (Ashamu, 2011 ; Kamga, 2011).

Après l'exposé de quelques brefs éléments relatifs à la nature et à la forme du droit au développement en droit international (1.), il est donc

2 "Quel auteur peut encore invoquer le droit au développement sans devoir faire l'objet du reproche infamant de "tiers-mondiste démodé" ? " (Kohen, 2001).

3 Pour une fiche de présentation de la dernière affaire d'importance, une synthèse du GITPA sur Peuple Endoroi vs. Kenya : http://www.gitpa.org/Autochtone\%20 GITPA\%20300/GITPA300-153\%20ACCES\%20\%20JUSTICE\%20ENDOROI\% 20. htm (avril 2016). 
proposé dans le cadre restreint de cette étude une approche commentée de cette jurisprudence relativement méconnue, en abordant ensuite les prémices juridictionnelles (2.), tâtonnements préalables à la formulation d'une décision de principe (Endorois c. Kenya) adoptée le 25 novembre 2009, qui définit des axes directeurs (3.) pour un droit au développement qui n'est plus seulement en quête de reconnaissance, mais qui a atteint le stade de sa mise en application, entraînant toutes les questions afférentes à cet enjeu.

\section{Notion de droit au développement en droit international}

Il est nécessaire de bien distinguer le droit international du développement, tel qu'il a été imaginé après la décolonisation, du droit au développement proclamé au niveau universel (1.1.), avant d'évoquer son intégration dans le droit africain des droits de l'homme (1.2.).

\subsection{La distinction entre droit du développement et droit au développement}

Le droit au développement est souvent présenté comme une création originale et relativement floue, issue de l'imagination fertile d'un juriste sénégalais de fameuse mémoire, feu Kéba Mbaye $(1972)^{4}$. Considéré à la fois comme un droit de l'homme et comme un droit de l'État (Israël, 1983), il a longtemps été associé au projet de Nouvel ordre économique international (ONU, 1974a) cher aux non-alignés et sa conception était initialement marquée par les préoccupations tiers-mondistes ${ }^{5}$ des années 1970-1980 (ONU, 1974b). Un contenu polymorphe formulé au gré d'échanges doctrinaux et de résolutions des Nations unies plus ou moins consensuelles ${ }^{6}$ fit de ce droit une hypothèse

4 La première mention du droit au développement est le fait du père Louis-Joseph Lebret, coordinateur du premier Plan du Sénégal indépendant, dans son article "La vocation des peuples au développement " paru lors des Semaines sociales de France de 1959. La première affirmation universelle de ce droit est inscrite dans I'encyclique Populorum Progressio du pape Paul VI en 1967. Néanmoins, le mérite revient au juge Kéba Mbaye d'en avoir dressé un premier portrait juridique hors du cercle ecclésial ou de celui des économistes du développement.

5 Ces préoccupations qui paraissaient datées ont repris une certaine vigueur avec la crise économique et financière de 2008-2009 (ONU, 2012).

6 La Déclaration sur le droit au développement adoptée par l'Assemblée générale des Nations unies en 1986 avait rencontré l'opposition des États-Unis et l'abstention de huit autres États, dont le Royaume-Uni. II fallut attendre la Déclaration de Vienne de 1993 suite à la Conférence mondiale sur les droits de l'homme pour que la notion de droit au développement soit formellement acceptée par tous. 
dont la mise en ouvre pouvait apparaître comme une gageure, en l'absence de tout texte international universel à valeur contraignante. Le statut juridique du droit au développement est ainsi resté une question permanente, à l'instar du droit international du développement - expression forgée par André Philip lors de la première Cnuced en 1965 (Feuer, 1993) et reprise par Michel Virally (1965) -, lequel désigne l'ensemble des règles objectives visant à promouvoir, organiser et soutenir le processus de développement dans les pays dits du Sud. Le droit du développement, bien qu'il ait éprouvé quelques difficultés à trouver sa place en tant que corpus de normes s'éloignant du droit international classique ${ }^{7}$, est donc à distinguer du droit au développement, lequel désigne un droit subjectif, appartenant à une personne, de voir se réaliser à son bénéfice des obligations de développement dont elle est créancière auprès d'un débiteur déterminé (Hugon, 2005). En langue anglaise, la distinction est claire puisqu'on parle de law pour le droit objectif et de right pour un droit subjectif. Au fil de l'affirmation du caractère subjectif du droit au développement, celui-ci s'est détaché du seul rôle de fondement principiel du droit international du développement (Flory, 1977 ; Pellet, 1987 ; Bouveresse, 1990) qui lui avait d'abord été assigné,

Le droit au développement paraît bien faible car il se voit cantonné dans un rôle d'incitation politique, voire à une simple incantation de préambule dont la réalité juridique serait de ce fait fort douteuse. pour rejoindre le champ plus vaste de la théorie des droits de l'homme - polyvalente et non plus réduite à la sphère économique. Et si de nouveaux champs de recherche sont aujourd'hui ouverts à travers la conception émergente d'un droit international axé sur le développement et la reconnaissance (Tourme-Jouannet, 2011), le droit au développement peine encore à sortir de l'ordre moral pour entrer dans le siècle.

De surcroît, les commentateurs (de Decker, 1996 ; Tchakoua, 1996) estimaient difficilement concevable que le droit au développement puisse

7 Par la volonté, notamment, d'établir une dualité des normes en faveur des États en développement. Ceci a trouvé un certain écho en droit international économique avec le système de préférences généralisées adopté par le Gatt et l'OMC. 
avoir, un jour, une existence contentieuse ${ }^{8}$. Ici revient l'interrogation prégnante lancée dans un article mémorable : «Droits des pauvres, pauvre(s) droit(s) ? (Imbert, 1989). Ce droit impétrant paraît bien faible car, malgré son importance (ne fut-il pas qualifié de « norme métajuridique » (Sanson, 1984) et d'« alpha et oméga des droits de l'homme » (Bejaoui, 1991) ?), il se voit cantonné dans un rôle d'incitation politique, voire à une simple incantation de préambule dont la réalité juridique serait de ce fait fort douteuse. Car est-il vraiment question d'un droit opposable lorsqu'il ne peut y avoir de contentieux dans les faits? Ce n'est certes pas le seul critère de l'opposabilité d'un droit subjectif, mais l'invocation d'une norme devant les prétoires et son existence dans les motifs de la jurisprudence témoignent d'une mutation réussie d'un postulat de l'opinio juris - c'est-à-dire la croyance pour les théoriciens et les praticiens qu'ils sont bien face à du droit, leur adhésion psychologique en somme - vers une substance juridique accessible pour le justiciable - puisqu'elle est reconnue par celui qui dit le droit, le juge. Tel n'était pas, il est vrai, l'objectif premier des fondateurs du droit au développement, lesquels ont établi une vision collective de ce droit qui se ressent dans sa définition même :

Le droit au développement est un droit inaliénable en vertu duquel toute personne humaine et tous les peuples ont le droit de participer et de contribuer à un développement économique, social, culturel et politique dans lequel tous les droits de l'homme et toutes les libertés fondamentales puissent être pleinement réalisés, et de bénéficier de ce développement (ONU, 1986)9.

Élément souvent associé à un discours idéologiquement connoté, il a apparemment cédé la place à une dialectique néolibérale et, en nos temps de négociations climatiques, il est loisible de constater certaines insuffisances de la Déclaration sur le droit au développement de 1986 qui ne fait nulle mention de l'environnement. C'est l'ONU qui a maintenu ce débat vivant par l'intermédiaire de réunions de groupes de travail visant à approfondir le thème, mais l'audience desdites instances a été fort limitée jusqu'aux années 2000 (Rupanner \& Kerchmeier, 2011), de sorte que le droit

8 L'absence d'invocabilité (et par conséquent, de justiciabilité) du droit au développement est également reprise par Valère Eteka-Yemet (2000).

9 Le reproche peut être également fait à cette définition d'occulter l'aspect individuel que peut prendre ce droit, que d'autres textes associent au libre épanouissement de la personnalité (cf. I'article 9 de la Constitution béninoise du 11 décembre 1990). 
au développement s'est alors perdu progressivement « dans les limbes des espoirs déçus » (de Béchillon, 1997).

\subsection{L'intégration dans le droit africain des droits de l'homme}

Un tel constat était certes encore compréhensible il y a dix ans. Entretemps s'est développé un phénomène inédit et d'initiative africaine : l'élaboration d'une jurisprudence du droit au développement à partir de l'un des seuls textes internationaux à valeur contraignante qui le consacre, à savoir l'article 22 de la Charte africaine des droits de l'homme et des peuples. L'essor de la jurisprudence de l'article 22 est l'une des expressions de la spécificité de la Charte africaine, qui mêle des droits individuels et des droits collectifs dans un même instrument de protection, ce qui lui a valu des reproches d'ineffectivité et d'aporie. C'est

Organisme intergouvernemental prônant la conciliation, la Commission africaine des droits de l'homme et des peuples correspond, selon ses fondateurs, à l'esprit africain de la palabre pour résoudre les conflits. tout d'abord la carence institutionnelle qui est en cause (Mubiala, 2005) : à l'origine, seule la Commission ADHP existait. C'est elle qui reçoit les plaintes tant des individus que des groupes minoritaires ou autochtones, à l'issue de l'épuisement des voies de recours internes. Organisme intergouvernemental prônant la conciliation, elle correspond, selon ses fondateurs, à l'esprit africain de la palabre pour résoudre les conflits ${ }^{10}$. Surtout, cette quasi-juridiction était sans doute le seul procédé de contrôle acceptable pour de jeunes États ayant acquis depuis peu une souveraineté internationale. Une modernisation a eu lieu avec l'adoption du Protocole de 1998 instaurant une véritable Cour africaine des droits de l'homme et des peuples ${ }^{11}$; sise à Arusha (Tanzanie), cette juridiction

10 "Les conflits sont tranchés non pas par une procédure contentieuse mais par la conciliation [...] qui aboutit à un consensus et qui ne fait ni vainqueur ni vaincu " (Mbaye, 2002).

11 Protocole relatif à la Charte africaine des droits de l'homme et des peuples, portant création d'une Cour africaine des droits de l'homme et des peuples, adopté en juin 1998 à Ouagadougou. 
est désormais opérationnelle ${ }^{12}$. Si le nombre d'affaires en cours est certes sans commune mesure avec la situation de la Cour européenne des droits de l'homme ${ }^{13}$, il est indéniable que le système africain rend bel et bien des décisions ; il convient par ailleurs de souligner le rôle important qui y est joué par les organisations non gouvernementales (ONG) qui ont accès aux prétoires et peuvent faire acte de représentation des victimes de violations de la Charte (Debos, 2005). Les ONG sont d'ailleurs à l'origine d'une bonne part de la jurisprudence du droit au développement.

Quant à l'aporie normative alléguée, elle vient des réserves de certains juristes « gardiens du temple des droits de l'homme » (Mbaye, 1980) qui perçoivent les droits collectifs comme une menace pour les droits individuels (Haarscher, 1990). Il s'agirait de pseudo-droits dans les mains de l'État, et non de normes protectrices ${ }^{14}$. L'originalité de la Charte est d'associer l'ensemble de ces générations de droits en un même texte, aboutissant à un «métissage normatif » (Boukongou, 2006) : en reconnaissant formellement le droit au développement aux côtés des autres droits plus classiques, l'article 22 de la Charte africaine est un exemple d'intégration de normes nouvelles dans le corpus traditionnel. C'est dans cette optique que les commissaires et juges africains participent à travers leurs décisions à la clarification du droit au développement. Cette politique jurisprudentielle est un pivot conceptuel, car l'incidence statistique d'une accumulation de décisions - n'ayant certes que l'autorité de la chose jugée inter partes, mais dégageant des principes transposables aux cas similaires - est nécessaire pour passer du vœu à la norme (Langrognet, 2015) ${ }^{15}$.

12 Ce qui amène à une structure juridictionnelle comparable à celle qui était en place au sein du Conseil de l'Europe avant l'adoption du protocole n 11 du 11 mai 1994 supprimant la Commission européenne des droits de I'homme.

13 Laquelle compte des milliers d'affaires pendantes chaque année devant son prétoire.

14 Karel Vasak (1984) les a appelés droits « de troisième génération ». L'accueil critique a été mitigé dans la doctrine, en l'absence de textes contraignants puisqu'il s'agissait pour l'essentiel de recommandations des Nations unies. Il est usuel ces dernières années de ne pas prendre au sérieux ces droits (Dworkin, 1995), rabaissés au rang d'objectifs et de vœux hors du droit, trahissant une " langue de bois des droits de I'homme » (Sudre, 2012).

15 Concernant le rôle de la jurisprudence dans le renforcement de l'opposabilité d'une norme internationale, voir la Convention sur les droits de l'enfant de 1989. 


\section{Les patientes esquisses d'un droit mouvant à partir de l'an $\mathbf{2 0 0 0}$}

Ce n'est qu'à l'aube du nouveau millénaire que des requérants divers ont commencé à solliciter auprès des commissaires et juges internationaux africains la défense de leur droit au développement en tant que garantie subjective, imposant des devoirs exigibles à l'État. Le chemin entre reconnaissance implicite et défense explicite de ce droit a été parcouru en moins d'une décennie.

\subsection{Une justiciabilité associée à d'autres droits de l'homme, ou un droit au développement incident}

Quelques démarches, parfois balbutiantes, ont conduit la Commission africaine des droits de l'homme et des peuples à se prononcer sur un droit contesté dans son existence même. La Commission de Banjul a dès lors adopté une attitude certes prudente, mais également progressiste. L'affaire dite des Ogoni (Commission ADHP, 2001), dans laquelle des populations résidant dans le delta du Niger étaient victimes d'une pollution massive du fait d'une activité pétrolière intense et non régulée, en est un exemple typique. Si la Commission ne cesse, tout au long de son raisonnement, de revenir sur les conditions de vie dégradées des Ogoni à cause d'une industrie dont ils ne retirent aucun bénéfice, la violation de la Charte par le Nigeria est finalement retenue au titre de l'article 24, et non de l'article 22 . Il est vrai que la formulation de l'article 24, qui protège l'environnement, permet de déborder sur le développement : «Tous les peuples ont droit à un environnement sain et satisfaisant, propice à leur développement ». Tout au plus la Commission s'est-elle hasardée à avancer qu'il y avait une violation implicite du droit au développement à travers la pollution des moyens de subsistance des Ogoni. La Cour de justice de la Communauté économique des États de l'Afrique de l'Ouest a adopté un raisonnement quasi-identique dans son arrêt Serap c. Nigeria du 14 décembre $2012^{16}$ :

16 L'affaire portait sur des réclamations portées au nom de populations riveraines du delta du Niger, et victimes de la pollution de la région liée aux activités de la compagnie pétrolière Shell; on notera que les requérants avaient sollicité de la Cour qu'elle déclare dans son arrêt solennellement un ensemble de droits : "que toute personne dans le delta du Niger est titulaire d'un droit de I'homme internationalement reconnu à un niveau de vie adéquat, incluant un accès adéquat à la nourriture, à la santé, à une eau potable et à un environnement sain ; au développement économique et social ; et à un droit à la vie, à la sécurité humaine et à la dignité " (Cour 
Le cœur du litige est de déterminer si, dans les circonstances dont il s'agit, l'attitude de la République fédérale du Nigéria, en tant que Partie à la Charte africaine des droits de l'homme et des peuples, est en conformité avec les obligations souscrites à l'article 24 dudit instrument. [...] L'article 24 de la Charte requiert donc de tout État de prendre toute mesure pour maintenir la qualité de l'environnement compris comme un tout intégré, de sorte que l'état de l'environnement puisse satisfaire les êtres humains qui vivent là et accroître leur développement durable. ${ }^{17}$ (Cour de justice de la CEDEAO, 2012, §§ 98-101).

L'invocation de l'article 22 a d'abord été le fait de requêtes étatiques visant à protéger leur population en faisant condamner par la Commission des États limitrophes coupables de diverses exactions et ingérences sur leur territoire. C'est dans cet esprit qu'il faut lire la décision République démocratique du Congo c. Burundi, Ouganda et Rwanda (n²27/99) de mai 2003: «La dénégation, dans cette affaire, du droit des populations de la RDC de disposer librement de leurs richesses et de leurs ressources naturelles, a également causé une autre violation: leur droit au développement. » (Commission ADHP, 2003a, §§ 94-95). La Commission a néanmoins encore fait preuve de prudence, puisqu'elle associe l'article 22 à la violation de l'article 21 (droit des peuples à disposer de leurs ressources naturelles ; en l'espèce, les provinces de l'Est congolais étaient occupées et

La justiciabilité tient du défi lorsque I'on passe du stade des obligations négatives à celui des obligations positives. Ce passage constitue la difficulté majeure à laquelle sont confrontés les droits économiques et sociaux, a fortiori le droit au développement. pillées par les États voisins) pour reconnaître la violation du droit au développement des habitants de ces régions, ce dernier étant manifestement réduit à néant (Commission ADHP, 2003a).

\subsection{L'émergence contentieuse propre de l'article 22 de la Charte africaine}

Le glissement de l'article 22 vers une véritable justiciabilité pour les personnes humaines est à mettre en relation avec la réflexion initiée par la Commission sur la situation et les droits des peuples autochtones et

de justice de la Cedeao, 2012, § 19, traduction de l'auteur). La Cour n'a pas suivi cette énumération qu'elle a considéré trop éloignée de l'affaire qui lui était soumise, et n'a constaté que la violation des articles $1^{\text {er }}$ et 24 de la Charte africaine.

17 Traduction de l'auteur. 
minoritaires, objet de plusieurs résolutions depuis 2003 (Commission ADHP, 2003 b). La « justiciabilité » offre une conception large de l'action du juge par rapport à l'invocabilité simple telle qu'elle est entendue d'ordinaire : c'est non seulement la possibilité formelle pour un magistrat de connaître d'une réclamation concernant un droit et d'annuler l'acte par lequel l'État s'est exagérément immiscé dans les libertés individuelles, mais surtout il s'agit de la capacité intrinsèque de la règle d'être garantie et réalisée sur injonctions prétoriennes (Roman, 2012) : en droit international, c'est la concrétisation de ce que certains États, tels les États-Unis, ne considèrent pas comme du droit, mais simplement comme des « objectifs ambitieux » et surtout, non contraignants. La justiciabilité tient du défi dès qu’on dépasse le stade des obligations négatives (qui exigent une abstention de l'État, concernant pour l'essentiel la première génération « classique » de droits de l'homme, que le défunt bloc socialiste appelait « libertés formelles ») et que l'on s'aventure sur le terrain des obligations positives (qui supposent une action de l'État, souvent le cas des droits de l'homme de seconde et de troisième génération, les «droits réels »). Le passage de cette épreuve juridictionnelle constitue la difficulté majeure à laquelle sont confrontés les droits économiques et sociaux $^{18}$, a fortiori le droit au développement.

Le piétinement de tout droit au développement d'une minorité est ainsi la cause d'une véritable philippique de la Commission contre l'État soudanais dans la décision Organisation soudanaise des droits de l'homme \& C.O.H.R.E c. Soudan du 27 mai 2009 (Commission ADHP, 2009a). L'affaire concerne l'oppression des communautés noires du Darfour ${ }^{19}$ et la Commission a dressé un tableau sévère de ce que l'article 22 exigeait en l'espèce, indiquant même la politique à suivre par l'État au regard du droit :

La revendication de traitement égal par le peuple du Darfour est issue du sous-développement et de la marginalisation dont souffre la région. C'est à

18 « Le juge peut refuser sa compétence limitée vis-à-vis d'un droit sur le fondement de son caractère impropre au contrôle juridictionnel [...] L'affirmation de leur incapacité est souvent étayée par le refus du juge de définir une obligation juridique sur le fondement de la disposition contenant le droit. " (Nivard, 2009).

19 La Commission se lance d'ailleurs à travers ce dispositif dans une définition du " peuple " au sens de la Charte, à partir de critères ethniques, sociaux et culturels, lesdits critères étant confirmés par une identification propre qui est l'œuvre tant par les personnes concernées que par les personnes extérieures au groupe visé. 
cet égard que la Commission conclut à une violation de l'article $22:[\ldots]$ les attaques, menées tant par les forces du Gouvernement que par leurs alliés, les milices janjaouites, et les déplacements forcés de population du Darfour leur ont dénié la possibilité de s'engager dans des activités économiques, sociales et culturelles. Les déplacements entravent le droit à l'éducation de leurs enfants et la poursuite d'autres activités. Au lieu de déployer ses ressources pour résoudre le problème de la marginalisation de cette région pauvre et isolée, qui était la principale source du conflit, l'État défendeur a lancé une campagne militaire punitive qui constitue une violation massive de tous les droits de l'homme. (Commission ADHP, 2009a).

Les deux aspects du droit au développement se dessinent dans cette décision : d'une part, la liberté de se développer qui requiert l'élimination des obstacles au développement, notamment en respectant les autres droits de l'homme ${ }^{2 \mathrm{O}}$ - c'est « la possibilité de s'engager dans des activités... »-, et, d'autre part, l'exigence de progrès économique et social qui demande l'intervention des pouvoirs publics pour améliorer la situation de leurs administrés ${ }^{21}$, c'està-dire une obligation d'élaborer des projets de développement au bénéfice de la population.

Les deux aspects du droit au développement sont : la liberté de se développer qui requiert l'élimination des obstacles au développement, et l'exigence de progrès économique et social qui demande l'intervention des pouvoirs publics.

À l'inverse, dans une décision adoptée à la même session de novembre 2009, l'affaire Bakweri Land Claim c. Cameroun, la Commission - confirmant un raisonnement qu'elle avait déjà tenu six mois auparavant dans sa décision Kevin Mgwanga et al. c. Cameroun (Commission ADHP, 2009b) - n'a pas retenu contre l'État défendeur de violation de l'article 22, considérant que les ressources insuffisantes des États en développement ne permettent pas une répartition homogène des investissements publics sur le territoire. La bonne volonté de l'État dans l'affectation des ressources entre

20 Ici la théorie du droit au développement rejoint certaines thèses économiques, et notamment celle d'Amyarta Sen mesurant le développement à l'aune de l'augmentation des " capabilités ".

21 Autrement dit, la couverture toujours améliorée des "coûts de l'homme " de François Perroux (1991). 
les différents échelons locaux est ici étayée par des instruments statistiques. La Commission a alors exprimé sa compréhension vis-à-vis du « grand défi que constitue pour l'État la réalisation du droit au développement »: d'aucuns verraient sans doute dans ces mots la révélation contrite de la faible justiciabilité de ce droit par les commissaires eux-mêmes. Ce n'est pourtant pas le sens à attribuer à cette décision où, fait remarquable, une instance quasi juridictionnelle internationale procède à l'examen d'une politique de développement d'un État, avec l'accord de celui-ci. De décision en décision, la Commission africaine a progressivement avancé sur un terrain difficile faisant partie des prérogatives souveraines des États, à savoir les politiques publiques de développement ${ }^{22}$. Dans les faits, reconnaître la difficulté de la réalisation du droit au développement n'empêche pas d'établir sa validité en tant qu'obligation de moyen à la charge des autorités publiques. Ce type d'obligation induit une définition quelque peu conditionnée, et pour tout dire quasi fonctionnelle $e^{23}$, de la souveraineté étatique qui n'est plus aussi absolue qu'autrefois dans une société internationale marquée par l'affermissement de normes communes d'effet direct (Chrestia, 1999) et d'objectifs globalisants ${ }^{24}$. L'effet de convergence peut en être positif, mais les risques de déstabilisation de l'ordre en place, même au nom du développement humain, ne sont pas à négliger ${ }^{25}$. C'est pourquoi le gardien de la Charte africaine entend ici borner sa compétence quant aux violations de l'article 22 vis-à-vis des ruptures d'égalité entre les différents groupes et ethnies à l'intérieur d'un État : cette autolimitation s'explique par la faible marge de manœuvre de la Commission dans son activité juridictionnelle, l'organe international ne pouvant se permettre d'être accusé de juger en opportunité sur un sujet aussi vaste que le développement. La décision relative aux Endorois, adoptée fin 2009 et publiée en 2010, a approfondi et amplifié ces premières approches crescendo dans un dispositif qui marque la véritable naissance du droit au développement.

22 Un terrain sur lequel les États en développement sont d'autant plus jaloux de leur liberté d'action qu'ils considèrent leurs choix de développement comme l'expression souveraine du droit des peuples à disposer d'eux-mêmes.

23 Et dont on retrouve des éléments de définition dans la thématique contemporaine de la "responsabilité de protéger".

24 Les Objectifs du millénaire pour le développement, auxquels viennent de succéder les Objectifs de développement durable, en sont autant d'exemples.

25 Bien que l'idée de souveraineté conditionnelle ait déjà été émise en des temps anciens par Grotius (Fierens, 2014). 


\section{L'apport essentiel d'une décision historique : Endorois c. Kenya (2009)}

Cette décision a replacé le droit au développement au centre des préoccupations du droit international en Afrique ${ }^{26}$ et a mis en place un protocole éthique permettant de le structurer en tant que droit subjectif tendant vers la catégorie des droits fondamentaux. La fondamentalité est un concept juridique (Coudert, 2011) qui s'est développé avec vigueur ces dernières années, attribuant aux droits fondamentaux une fonction de vecteurs de valeurs et de légitimité, capables de modifier ou d'orienter la substance du droit positif. L'exemple le plus parlant se trouve dans les droits de l'homme, dont l'application transcende les divisions catégorielles : il s'agit d'objets juridiques dont la nature singulière se distingue au sein de l'ensemble du droit objectif, et qui ne s'épuisent pas dans le droit positif en vigueur (Picard, 1998). C'est en ce sens que l'ONU l'affirme comme un droit « inaliénable »(ONU, 1986) de la personne humaine, sous-entendant par là que c'est Les droits de l'homme sont des objets juridiques dont la nature singulière se distingue au sein de l'ensemble du droit objectif, et qui ne s'épuisent pas dans le droit positif en vigueur.

à l'ordre positif de s'adapter au droit subjectif, et non l'inverse. Le système africain des droits de l'homme participe à ce processus en jaugeant les pratiques nationales à l'aune de ce droit dans les requêtes qui lui sont soumises.

\subsection{La formulation juridique d'une éthique du processus de développement}

C'est dans le cadre de l'application d'un projet de développement que la Commission de Banjul a adopté la décision Endorois c. Kenya (Commission $\mathrm{ADHP}, 2009 \mathrm{c})$, qui a rendu le droit au développement justiciable dans le système africain. En l'espèce, l'État kényan avait expulsé dans les années 1970 des tribus d'éleveurs proches des Masaï, les Endorois, de leurs terres ancestrales situées dans la province de la Vallée du Rift pour y aménager une réserve faunique et des complexes hôteliers bâtis sur les rives du lac Bogoria. En

26 En témoigne le renouveau des études doctrinales en langue anglaise sur ce sujet depuis cette date. 
conséquence, les Endorois avaient été refoulés avec leurs troupeaux vers des terres ingrates et ne pouvaient plus accéder à ce lac bordé de montagnes et de geysers pour leurs cérémonies rituelles. En contrepartie de cette aliénation, l'État défendeur ne leur avait octroyé qu'une compensation ponctuelle symbolique et leur interdisait l'accès à la réserve, pourtant constituée sur leur patrimoine tribal collectif, titre de propriété que les juridictions kényanes s'étaient refusé à reconnaître tout au long de la procédure interne. À ces critiques, l'État kényan rétorquait en utilisant le motif d'intérêt général : "La tâche des communautés dans une démocratie participative est de contribuer au bien-être de la société dans son ensemble et non seulement de veiller sur sa propre communauté au détriment d'autres. »(Commission ADHP, 2009c). Incidem-

Si le développement est une mission politique du gouvernement et que l'élection constitue l'approbation populaire du programme ou du bilan, l'arrivée au pouvoir d'une majorité démocratique ne suffit pas en soi à garantir l'accomplissement par l'État de ses devoirs de développement. ment, cela revient à nier la possibilité d'ayants droit (au développement) autres que la nation dans son ensemble ; le seul moyen d'expression de ce droit serait alors le vote politique, et non la possibilité d'un contrôle juridictionnel. Il est vrai que prima facie, le développement est par excellence une mission politique du gouvernement et que l'élection constitue l'approbation populaire du programme ou du bilan dans ce domaine. Mais cela ne vaut pas quitus en droit, car l'arrivée au pouvoir d'une majorité démocratique ne suffit pas en soi à garantir l'accomplissement par l'État de ses devoirs de développement. La Commission n'a donc pas, en toute logique, soutenu cette vision des choses qui relègue le développement dans le discrétionnaire étatique. Un paragraphe de principe restera sans doute dans les annales de sa jurisprudence : « Il incombe à l'État défendeur la responsabilité27 de créer les conditions favorables au développement des peuples. [...] L'État défendeur a l'obligation de garantir aux Endorois qu'ils ne sont pas exclus du processus de développement ou du partage des bénéfices. » (Commission ADHP, 2009c, § 298).

27 L'emphase est de l'auteur : qui dit « responsabilité " dit reddition des comptes sinon à des ayants droit, du moins à des requérants ayant qualité à agir pour mettre en cause cette responsabilité. 
On comprend dès lors le retentissement dans les cénacles internationaux de cette décision, saluée même par le Haut-commissaire des Nations unies aux droits de l'homme. En se fondant sur de nombreux éléments de droit comparé - notamment la Déclaration sur le droit au développement du 4 décembre 1986 et la jurisprudence de la Cour interaméricaine des droits de l'homme -, la Commission conclut à la violation de l'article 22 de la Charte et définit le droit qui y est inscrit comme un phénomène avec une double opposabilité, à la fois formaliste et finaliste. Cette jurisprudence envisage ainsi le développement - et le droit subjectif qui y est associé - en deux volets complémentaires : l'un sur la procédure, l'autre sur le fond, chacun devant être également respecté. La Commission identifie ensuite cinq critères exigibles : le droit au développement requiert une action publique « équitable, non-discriminatoire, participative, responsable et transparente », rejoignant le thème bien connu de la bonne gouvernance ; elle ajoute que sa réalisation doit refléter « l'équité et la possibilité de choisir, des thèmes importants et indispensables du droit au développement. » (Commission $\mathrm{ADHP}, 2009 \mathrm{c}, \S 277)$.

\subsection{Un droit au développement plus lisible et rénové ${ }^{28}$}

La décision relative aux Endorois a eu le mérite de souligner, d'un point de vue pratique, l'importance de la participation au développement, à la fois en tant que mode de consultation apportant une connaissance libre et éclairée des intéressés. En l'espèce, cela n'avait pas été respecté : l'État s'était contenté de transmettre des informations partielles à certains chefs de tribus

La participation au développement prévoit une connaissance libre et éclairée des intéressés ainsi qu'une répartition équitable des bénéfices issus du processus de développement auxquels les êtres humains ont droit. (Commission ADHP, 2009c,

$\S 292)$. De plus, la participation doit être comprise en tant que répartition équitable des bénéfices issus du processus de développement, auxquels les êtres humains, ici les Endorois, ont droit. Cette répartition se matérialise

28 Ce que la Commission africaine dénomme le " volet instrumental » (ou procédural) et le " volet substantif " (ou constitutif) du droit au développement. 
au moins par une juste indemnisation (Commission ADHP, 2009c, § 295) et des garanties permettant de préserver le mode de vie des populations locales troublé par l'intervention extérieure, au mieux par l'association de ces populations à la gestion du projet et par la jouissance équitablement répartie des fruits de l'expansion économique. En l'espèce, le Kenya n'avait fait ni l'un, ni l'autre (Commission ADHP, 2009c, § 297), alors que « le partage des retombées est la clé du processus de développement » ainsi qu'une obligation en vertu d'une autre convention internationale, la Charte africaine sur la participation et la transformation au développement de 1990. Trente-sept ans après leur expulsion, cette décision a permis le retour des Endorois sur leur terre en 2010 (AFP, 2010) ; elle constitue, sur le plan juridique, un jalon majeur du droit au développement en ce début de $\mathrm{XXI}^{\mathrm{e}}$ siècle. Il est à noter un prolongement remarquable de cette judiciarisation du droit au développement dans l'ordonnance de la Cour africaine des droits de l'homme et des peuples du 15 mars 2013 portant mesures provisoires dans un contentieux similaire, concernant l'expulsion de la communauté Ogiek du massif forestier de Mau dans l'est du Kenya (Cour ADHP, 2013). Sur le fondement d'un risque de violation du droit au développement de cette peuplade forestière, le juge international africain en vient à suspendre l'action de l'État : là aussi, l'effort jurisprudentiel en faveur du droit au développement est remarquable et semble répondre à ceux qui pensaient ce droit inexistant qu'il recouvre, in jure, une certaine réalité.

La Commission, qui est à l'origine de cette requête de mesures provisoires $^{29}$, a de son côté continué son effort en adoptant la résolution $\mathrm{n}^{\circ} 197$ du 5 novembre 2011 relative au processus d'inscription du lac Bogoria sur la liste du patrimoine mondial de l'Unesco, initié par le gouvernement kényan : «Ce processus de décision enclenché par les autorités administratives, sans la participation des Endorois, est une violation du droit au développement garanti par l'article 22 de la Charte africaine. » Insistant sur l'entière application de sa décision de 2009, la Commission a exhorté le Kenya, par sa résolution $\mathrm{n}^{\circ} 257$ du 5 novembre 2013, à lui adresser des rapports réguliers et une feuille de route sur la situation des Endorois : l'appel a été entendu

29 Cette saisine de la Cour, instance supérieure, est la preuve de l'intérêt que porte la Commission au contentieux relatif au sort des peuples autochtones dans les projets de développement, alors qu'elle-même a tout à fait la capacité de prendre des mesures provisoires de son propre chef. 
puisque, comme évoqué en introduction, l'État kényan a formé en septembre 2014 un groupe de travail chargé de mettre en œuvre les différents points de la décision, dont l'activité est suivie par les ONG concernées et la Commission (Minority Rights Group International, 2014).

Il est permis d'espérer que, de cette action volontariste et soutenue des juridictions et quasi-juridictions africaines, est en train d'émerger progressivement un véritable droit subjectif dont l'impact n'est pas marginal puisque ces décisions s'appliquent à des populations d'une certaine taille : plus de 60000 personnes pour les Endorois, et environ 30 ooo pour les Ogiek. Certes, l'œuvre n'est pas achevée : la question des ayants droit reste encore ambiguë, la Commission n'ayant traité que des requêtes collectives liées à l'article 22. Enfin, une autre faille du système africain des droits de l'homme, en comparaison de ce qui se passe en Europe, est la rare reprise des précédents régionaux dans les jurisprudences nationales. Ceci dit, il est indubitable que de tels progrès contentieux permettent à la notion de droit au développement de gagner en juridicité et, in fine, d'être mise en œuvre.

\section{Enguerrand SERRURIER}

Doctorant en droit public et Ater à l'université d'Auvergne (Clermont-Ferrand). enguerrand.serrurier@udamail.fr

\section{A récemment publié :}

Serrurier E., 2016, "Bref tableau des opportunités juridiques de la coopération franco-chinoise ", Pensées vives, Spécial Chine $n^{\circ} 1$, http://edlshs. univ-bpclermont.fr/sites/edlshs.univ-bpclermont.fr/IMG/pdf/PV_S1_Serrurier. pdf ( avril 2016).

Serrurier E., 2015, "Déclin, résistance et perspectives du droit français dans la compétition juridique mondiale ", Journal du Droit International (Clunet), n 2015/4, pp. 31-46.

Serrurier E., 2015, "Le développement dans le mandat du maintien de la paix: observations sur les missions au Darfour et au Soudan du Sud ", in Société française pour le droit internatonial, Droit international et développement, Colloque de Lyon (22-24 mai 2014), Paris, A. Pedone, pp. 281-290. 


\section{BIBLIOGRAPHIE}

Agence France Presse (AFP), 2010, "Kenya : expulsés en 1973, les Endorois de retour sur leurs terres ", JeuneAfrique.com, le 20 mars, http://www. jeuneafrique.com/depeches/91274/ politique/kenya-expulses-en-1973-lesendorois-de-retour-sur-leurs-terres/ (avril 2016).

Ashamu E., 2011, " Centre for Minority Rights Development (Kenya) and Minority Rights Group International on Behalf of Endorois Welfare Council v. Kenya: A Landmark Decision from the African Commission ", Journal of African Law, vol. 55, n², pp. 300-313, http://journals. cambridge.org/action/displayAbstract? fromPage $=$ online $\&$ aid $=8378407 \&$ filel $d=$ S0021855311000155.

de Béchillon D., 1997, Qu'est-ce qu'une règle de droit ?, Paris, Odile Jacob.

Bedjaoui M., 1991, " Le droit au développement ", in Bedjaoui M. (dir.), Droit international. Bilan et perspectives (tome 2), Paris, A. Pedone.

Boukongou J.-D., 2006, " The Appeal of the African System for Protecting Human Rights ", African Human Rights Law Journal, vol. 6, n² 2, pp. 268-298, http://www.ahrlj.up.ac.za/boukongou-j-d (avril 2016).

Bouveresse J., 1990, Droit et politique du développement et de la coopération, Paris, PUF.

Chrestia P., 1999, " L'influence des droits de l'homme sur le droit international contemporain ", Revue trimestrielle des droits de I'homme, n 40, pp. 715-738.
Commission ADHP, 2001, Serac lau nom des Ogoni) c. Nigeria, le 16 octobre, communication n¹55/96.

Commission ADHP, 2003a, République démocratique du Congo c. Burundi, Ouganda et Rwanda, le 29 mai, communication $n^{\circ} 227 / 99$.

Commission ADHP, 2003b, Résolution portant adoption du rapport $d u$ Groupe de travail sur les populations et communautés autochtones, ACHPR/ Res. 65 (XXXIV), le 20 novembre.

Commission ADHP, 2009a, Sudanese Human Rights Organization \& C.O.H.R.E. c. Soudan, le 27 mai, communications $n^{\circ} 279 / 05$ et $296 / 05$.

Commission ADHP, 2009b, Kevin Mgwanga et al. c. Cameroun, le 27 mai, n²66/2003, § 206.

Commission ADHP, 2009c, Centre for Minority Rights Development (on behalf of Endorois Welfare Council) $c$. Kenya, le 25 novembre, communication $\mathrm{n}^{\circ} 276 / 03$.

Coudert C., 2011, Réflexion sur le concept de fondamentalité en droit public français, Thèse de doctorat, ClermontFerrand, Université d'Auvergne.

Cour ADHP, 2013, Commission africaine des droits de l'homme et des peuples c. Kenya, ordonnance portant mesures provisoires, req. n006/2012, le 15 mars.

Cour de justice de la Cedeao, 2012, SocioEconomic Rights and Accountability Project (Serap) c. Federal Republic of 
Nigeria, ECW/CCJ/JUD/18/12, le14 décembre, §§ 98-101

Debos M., 2005, " La création de la Cour africaine des droits de l'homme et des peuples. Les dessous d'une ingénierie institutionnelle multicentrée ", Cultures \& Conflits, $n^{\circ} 60$, pp. 159-182, https:// conflits.revues.org/1934 (avril 2016).

de Decker H., 1996, "Le droit au développement n'est pas un droit au sens strict. Parce qu'il n'est pas exigible en justice devant une autorité compétente. ", in Maugenest D., Pougoue P.-G. (dir.), Droits de l'homme en Afrique centrale, Colloque de Yaoundé 19-11 novembre 1994), Paris, Karthala.

Donnelly J., 1985, " In Search of the Unicorn. The Jurisprudence and Politics of the Right to Development ", California Western International Law Journal, vol. 15, n³, pp. 473-509.

Dworkin R., 1995, Prendre les droits au sérieux, Paris, PUF.

Eteka-Yémet V., 2000, La Charte africaine des droits de l'homme et des peuples: étude comparative, Paris, L'Harmattan.

Feuer G., 1993, " Le droit international du développement. Une création de la pensée francophone ", in Choquet C. et al., État des savoirs sur le développement, trois décennies de sciences sociales en langue française, Paris, Karthala, pp. 87-96.

Fierens J., 2014, Le droit naturel pour le meilleur et pour le pire, Namur, Presses universitaires de Namur.

Flory M., 1977, Droit international du développement, Paris, PUF.
Haarscher G., 1990, "Les droits collectifs contre les droits de l'homme ", Revue trimestrielle des droits de l'homme, $1990, n^{\circ} 3$.

Hugon P., 2005 " Droit, droits et économie du développement ", Mondes en développement, $\mathrm{n}^{\circ} 129, \mathrm{pp} .13-40$, http://www.cairn.info/revue-mondesen-developpement-2005-1-page-13.htm (avril 2016).

Imbert P.-H., 1989, " Droits des pauvres, pauvre(s) droit(s) ? Réflexion sur les droits économiquesn sociaux et culturels ", Revue de droit public et de la science politique en France et à l'étranger, n³, pp. 739-754.

Israël J.-J., 1983, " Le droit au développement ", Revue générale de droit international public, pp. 5-41.

Kamga S., 2011, "The Right to Development in the African Human Rights System: The Endorois Case ", De Jure, vol. 44, n² 2, pp. 381-391, http://www.dejure.up.ac.za/index.php/ volumes/44-vol-2-2011/articles/kamgasad (avril 2016).

Kohen M. G., 2001, " Manifeste pour le droit international du XXle siècle ", in Boisson de Chazournes L., Gowlland V. (ed.), The International Legal System in Quest of Equity and Universality. Liber Amicorum Georges Abi-Saab, La Haye, Kluwer, pp. 123-152.

Langrognet F., 2015, " De l'incantation à la norme : I'incidence statistique croissante de l'intérêt supérieur de l'enfant dans le contentieux de l'éloignement des étrangers ", La revue des droits de l'homme, $n^{\circ} 7$, https://revdh.revues. org/1099 (avril 2016). 
Mbaye K., 1972, "Le droit au développement comme droit de l'homme. Leçon inaugurale à l'Institut international des droits de l'homme René Cassin (Strasbourg) ", La revue des droits de I'homme, vol. 5, n 2-3, pp. 505-534.

Mbaye K., 1980, "Le droit au développement ", Ethiopiques, n 21, http:// ethiopiques.refer.sn/spip.php?article736 (avril 2016).

Mbaye K., 2002, Les droits de I'homme en Afrique, Paris, A. Pedone.

Minority Rights Group International, 2014, "Kenyan Task Force formed to implement the 2010 Endorois ruling ", minorityrights.org, le 29 septembre, http:// minorityrights.org/2014/09/29/kenyantask-force-formed-to-implement-the2010-endorois-ruling/ (avril 2016).

Mubiala M., 2005, Le système régional africain de protection des droits de I'homme, Bruxelles, Bruylant.

Nivard C., 2009, La justiciabilité des droits sociaux. Étude de droit conventionnel, Thèse de doctorat, Montpellier, Université de Montpellier.

ONU, 1974a, Résolutions de l'Assemblée générale $n^{\circ} 3201$ (S-VI) et n 3202 (S-VI) concernant l'instauration d'un nouvel ordre économique international, NewYork, ONU, le 1er mai, http://legal. un.org/avl/pdf/ha/ga_3201/ga_3201_ ph_f.pdf (avril 2016).

ONU, 1974b, Résolution de l'Assemblée générale $n^{\circ} 3281$ (XXIX) portant Charte des droits et devoirs économiques des États, New-York, ONU, le 14 décembre, http://www.un.org/french/ documents/view_doc.asp?symbol=A/ RES/3281 (XXIX)\&Lang=F (avril 2016).
ONU, 1986, "Article 1er ", Déclaration de l'Assemblée générale sur le droit au développement, New-York, ONU, le 4 décembre, http://www.un.org/fr/ events/righttodevelopment/declaration. shtml (avril 2016).

ONU, 2012, Résolution de l'Assemblée générale vers un nouvel ordre économique international, A/RES/67/217, New-York, ONU, le 21 décembre, http:// www.un.org/fr/documents/view_doc. asp? symbol=A/RES/67/217 (avril 2016).

OUA, 1981, Charte africaine des droits de l'homme et des peuples, Banjul, OUA, http://www.achpr.org/files/instruments/achpr/achpr_instr_charter_fra. pdf (avril 2016).

Pellet A, 1987, Le droit international du développement, Paris, PUF, coll. "Que sais-je ?".

Perroux F., 1991, L'Économie du xx siècle, Grenoble, Presses universitaires de Grenoble.

Picard E., 1998, "L'émergence des droits fondamentaux en France ", L'actualité juridique. Droit administratif, $n^{\circ}$ spécial : Les droits fondamentaux, une nouvelle catégorie juridique?, pp. 6-42.

Presidency of Kenya, 2014, " Task Force on the Implementation of the Decision of African Commission on Human and Peoples' Rights Contained in Communication n²76/03 ", Kenya Gazette, Notice $n^{\circ} 6708$, p. 2550, http://kenyalaw.org/kenya_gazette/gazette/volume/ MTA3Nw-Nol.CXVI-No.115 (avril 2016).

Renaudot T., 1632, "Relations des nouvelles du monde reçues tout le mois de mars 1632 ", La Gazette. 
Roman D., 2012, " La justiciabilité des droits sociaux ou les enjeux de l'édification d'un État de droit social ", La revue des droits de l'homme, $\mathrm{n}^{\circ}$ 1, https:// revdh.revues.org/635 (avril 2016).

Ruppanner V., Kirchmeier F., 2011, "Le droit au développement, un outil méconnu ", Le Temps, le 21 avril.

Sanson H., 1984, "Le droit au développement comme norme métajuridique en droit du développement ", in Flory $M$., Henry J.-R., Mahiou A. (dir.), La Formation des normes en droit international du développement. Actes du colloque d'Aix-en-Provence (7-8 octobre 1982), Paris, CNRS-OPU, pp. 61-70.

Santulli C., 2000, "Qu'est-ce qu'une juridiction internationale? Des organes répressifs internationaux à I'ORD ", Annuaire français de droit international, vol. 46, no 1, pp. 58-81, http://www. persee.fr/doc/afdi_0066-3085_2000_ num_46_1_3606 (avril 2016).

Sudre F., 2012, Droit européen et international des droits de l'homme, Paris, PUF.
Tchakoua J.-M., 1996, " Si le droit au développement n'est pas justiciable, c'est le fait des politiques. Volontairement ou non, ils ont prévu un droit non justiciable ", in Maugenest D., Pougoue P.-G. (dir.), Droits de I'homme en Afrique centrale. Colloque de Yaoundé (9-11 novembre 1994), Paris, Karthala.

Tourme-Jouannet E., 2011, Qu'est-ce qu'une société internationale juste? Le droit international entre développement et reconnaissance, Paris, A. Pedone.

Vasak K., 1984, "Pour une troisième génération des droits de I'homme ", in Swinarski C. (dir.), Études et essais sur le droit international humanitaire et sur les principes de la Croix-Rouge en I'honneur de Jean Pictet, Leyde, Martinus Nijhoff Publishers, pp. 837-845.

Virally M., 1965, "Vers un droit international du développement ", Annuaire français de droit international, vol. 11, $n^{\circ} 1$, pp. 3-12, http://www.persee.fr/doc/ afdi_0066-3085_1965_num_11_1_1805 (avril 2016). 